\title{
LAS CAJAS RURALES COMO PRIMER INTERMEDIARIO DEL SECTOR AGRARIO ANDALUZ
}

Eduardo SANCHEZ ANTONIJUAN*

\section{INTRODUCCION}

El objeto del presente trabajo no es tanto cuantificar la importancia de las Cajas Rurales en el sistema financiero andaluz y por ende en el sector agrario, ya que lo proporcionaría un simple cálculo de porcentajes sobre las estadísticas facilitadas por el Banco de España, sino poner de manifiesto el papel cualitativo y cuantitativo que están jugando en el campo andaluz.

Es de rigor, pues, empezar con una mera descripción del sector agrario andaluz y su importancia dentro de la economía andaluza y de la nacional, continuar con unos someros datos acerca de su financiación a través de todos los agentes del sistema financiero y de los agentes no financieros para, a continuación, entrar de lleno en la materia que nos ocupa, a saber: las Cajas Rurales con especial dedicación a las andaluzas.

Las fuentes consultadas han sido muy diversas, destacando el Instituto Nacional de Estadística a través del Censo Agrario de 1982, las Encuestas sobre la Población Activa... etc., el Ministerio de Agricultura y Pesca con sus inestimables Cuentas del Sector Agrario, los boletines y estadísticas del Banco de España, las memorias de las Entidades Oficiales de Crédito, el Plan Económico para Andalucía en sus Documentos, n. ${ }^{\circ} 2$ y n. 10 relativos al sector agrario y al sistema financiero respectivamente, la Memoria de la confederación de Cajas de Ahorro y los boletines estadísticos de la Junta de Andalucía. El resultado de tanta consulta ha sido, además de las siguientes líneas, contrastar de nuevo las discrepancias existentes entre las diferentes fuentes estadísticas consultadas.

Por último, no quería pasar por alto sin antes mencionar el asesoramiento y las opiniones de Cristina Roquero. Directora General de Política Financiera de la Junta de Andalucía a la sazón.

* Economista. Consejería de Economía. 


\section{EL SECTOR AGRARIO ANDALUZ EN EL CONTEXTO DE LA ECONOMIA REGIONAL}

Andalucía representa el 17,29\% de la superficie total de España y tient el $17,1 \%$ de la población aportando, sin embargo, el 12,59\% al P.I.B.

Estas tres cifras dicen por sí solas la importancia de esta Comunidac Autónoma en el contexto nacional. Ahora bien, si desglosamos esas tres ma. cromagnitudes nos encontramos que el $56,2 \%$ de las tierras son de cultivc incluyendo prados y pastizales, frente al $53,8 \%$ del total de España; que la población ocupada en el sector agrario es el 20,2\%, del que el $89,59 \%$ son varones; y que su aportación al P.I.B. agrario español es el 24,60\% represen. tando el $12,06 \%$ del P.I.B. andaluz frente al $6 \%$ que significa el sector agrario en el conjunto del P.I.B. nacional; lo que ilustra la aparente paradoja de la baja contribución de Andalucía al P.I.B. español.

En efecto, la economía agraria andaluza ha tenido siempre un papel predominante en la evolución de la economía de Andalucía. La no moderniza. ción de la agricultura a finales del siglo pasado y principios de éste implicó el paso de una economía diversificada, equilibrada, con grandes disponibilidades líquidas y capitales inversores a un retraso económico con una fuerte caida del empleo y abundantes crisis sectoriales.

Lamentablemente, quizás por la ausencia de financiación adecuada, no ha tenido aquí el mediano empresario la capacidad de empuje necesaria para modificar el curso de los acontecimientos. Así, ese 35,53\% de número de explotaciones con una extensión utilizada no ha servido para dinamizar a ese otro $0,18 \%$ de explotaciones con extensiones superiores a $500 \mathrm{Ha}$. que proveen el $14,55 \%$ de la superficie y sólo la utilizan en un $61,67 \%$ y viceversa.

En este sentido, cobra especial relevancia la estructura y configuración empresarial de los agentes productores. En Andalucía, la mayor parte de la producción es generada por empresarios individuales, con la excepción de las explotaciones superiores a las $1.000 \mathrm{Ha}$. en las que las sociedades mercantiles, sin llegar a igualar al número de empresarios individuales, adquieren cierto significado. Como ejemplo, podemos citar las explotaciones con extensiones comprendidas entre $3 \mathrm{Ha}$. y $30 \mathrm{Ha}$. en las que los empresarios individuales gestionan el $99,74 \%$ de las mismas $\mathrm{y}$, las mencionadas superiores a las 1.000 $\mathrm{Ha}$., en las cuales el número de empresarios individuales se reduce a casi la mitad -el 48,74\%-. El resto de fórmulas, asociativas o no, susceptibles de ser agentes productores carecen de importancia apreciable dentro del conjunto. Así, la gestión municipal o por cualquier entidad pública sólo adquiere signigicado -el 28,32 \% - a partir de las explotaciones con una superficie superior a las $500 \mathrm{Ha}$, la explotación comunal apenas si existe, las Sociedades Agrarias de Transformación representan tán sólo el 7,64\% dentro del conjunto total y las cooperativas están limitadas al 1,71\% . 
Llegados a este punto, lo que cabe preguntarse es si las explotaciones entre $3 \mathrm{Ha}$ y $30 \mathrm{Ha}$. son adecuadas en tamaño y rentabilidad para producir los productos típicos andaluces. En Andalucía destaca, es obvio, la producción de secano frente a la de regadío y se concentra básicamente en dos productos: los cereales y herbáceos, y el olivar. La producción de los primeros representa el 26,9 \% de la producción final agraria andaluza frente al $13 \%$ para el conjunto de España, en tanto que el olivar en Andalucía supone el $9,6 \%$ en la producción final frente al 2,6 \% del total nacional. Pues bien, las explotaciones cuyo tamaño estamos contemplando producen el $31,23 \%$ del total de ambos productos lo que junto a las explotaciones con superficie entre $30 \mathrm{Ha}$ y $200 \mathrm{Ha}$. -el 5,02\%-del número total de explotaciones significa producir el 69,29 \% de la producción final agraria andaluza.

Los estudios realizados en la campiña de secano de Sevilla muestran una rentabilidad media del 3,8\% en el período 1979-1981 en las explotaciones de tamaño entre $20 \mathrm{Ha}$. y $50 \mathrm{Ha}$, mientras que las explotaciones con superficie entre las $100 \mathrm{Ha}$. y $500 \mathrm{Ha}$. obtienen una rentabilidad del $0,2 \%$, es decir, prácticamente nula. No obstante, para el mismo período, la rentabilidad del arrendamiento ha sido del $8,75 \%$ lo que es perfectamente comparable con el rendimiento de cualquier otra inversión alternativa. No es de extrañar, por tanto, que el arrendamiento adquiera cierta importancia como régimen de explotación precisamente a partir de las $100 \mathrm{Ha}$. Efectivamente, el $90 \%$ de las explotaciones menores de $100 \mathrm{Ha}$. están cultivadas por sus propietarios disminuyendo esta proporción hasta el $77 \%$ a medida que el tamaño de la explotación aumenta a niveles entre $500 \mathrm{Ha}$ y $1.000 \mathrm{Ha}$, es decir, parace que existe una tendencia del agricultor a moverse dentro de las coordenadas del mercado y si se aleja no es por voluntad propia sino porque las condiciones externas no responden a sus necesidades.

En Andalucía, el grado de integración del sector agrario en la actividad económica global, es decir dentro de sus circuitos financieros, comerciales... etc., es muy elevado. Medido en términos de Valor Añadido Bruto en relación con la Producción final, en Andalucía alcanza el Valor del 69,5\% frente al $57,8 \%$ para el conjunto nacional, luego si en algún momento de la historia reciente ha habido una menor presencia del sector agrario en el devenir diario de la economía y de las finanzas regionales ha sido debido más a la falta de respuesta de las fuerzas económico-sociales que al desinterés del sector por transformarse. 


\section{LA FINANCIACION EN ANDALUCIA DEL SECTOR AGRARIO}

Analizando los medios financieros del sector vemos que las Entidade: Oficiales de Crédito financian directamente un $13 \%$, si bien los creditos in directos suponen un $9,6 \%$ del total de financiación al Sector, la Banca pri vada participa en un 27,86 \%, las Cajas de Ahorro en un 14,35\% y las Cajar Rurales en un $44,41 \%$. A niveles de cifras globales, la participación de los pósitos no es relevante aunque lo sea desde un punto de vista cualitativo pos la ausencia de tramitación del préstamo y por su bajo tipo de interés.

Comparando esta estructura financiera con el global a nivel nacional pare el sector agrario, encontramos que la Banca Oficial destina una mayor pro. porción de sus recursos hacia Andalucía que hacia otras regiones lo que es absolutamente coherente con el papel que la misma tiene asignado. La Banca privada, por su parte, también justifica su papel de intermediación captando los recursos donde los encuentra y transfiriéndolos hacia donde la rentabilidad de las inversiones es más óptima. Así en tanto que en Andalucía capta el $8,9 \%$ de sus recursos sólo invierte el $7,1 \%$ destinando el $7,36 \%$ al sector agrario. Este porcentaje se ha estimado extrapolando a Andalucía la estrategia bancaria seguida a nivel nacional. Es decir, si para el conjunto del país la Banca destina un $3,68 \%$ de sus colocaciones a la agricultura, este porcentaje se eleva en la misma medida que el sector agrario andaluz participa en el P.I.B. Obviamente, esta estimación no deja de ser un punto de partida optimista aunque la escasa relevancia del sector industrial en Andalucía y el nivel poco desarrollado de los servicios hacen posible la conjetura de que se produzcan simultáneamente los fenómenos de mayor dedicación al sector agrario al haber mayor demanda, y de transferencias a los otros sectores productivos estén situados estos dentro o fuera de la región. Las Cajas de Ahorro están perdiendo cuota de mercado sistemáticamente desde la O.M. del 31 de Enero de 1973 que suprimió la especialización agrícola en el coeficiente de préstamos de regulación especial y, en concreto, las Cajas Andaluzas han acelerado este proceso. No tiene ningún sentido que en Andalucía participen en un $14,35 \%$ en el total de financiación cuando a nivel nacional lo hacen en un $16,55 \%$, aunque este $14,35 \%$ signifique dedicar al sector casi el doble de atención que en otras regiones. Como luego veremos en el análisis de la estructura de los balances de los distintos tipos de entidades, las Cajas de Ahorros se han dedicado con harto pesar a la fácil colocación de sus recursos en la financiación del déficit sector público para luego hacer "gala" de la ausencia de dificultades mientras que la Banca y las Cooperativas de Crédito han atravesado malos momentos de solvencia. Las Cajas Rurales tienen en Andalucía un papel más importante que a nivel nacional ya que tanto en términos absolutos como relativos son el primer intermediario financiero del sector. 


\section{CUADRO I \\ FINANCIACION CREDITICIA AL SECTOR AGRARIO POR INSTITUCIONES PRESTAMISTAS AÑO 1984}

(Miles de millones de pesetas)

\begin{tabular}{|c|c|c|c|c|c|c|c|}
\hline \multirow{2}{*}{ Entidades } & \multicolumn{3}{|c|}{ ESPAÑA（A) } & \multicolumn{3}{|c|}{ ANDALUCIA (B) } & \multirow{2}{*}{$\begin{array}{c}\text { (B) } /(\mathrm{A}) \\
\%\end{array}$} \\
\hline & Miles mill. ptas. & Estructura & $\begin{array}{l}\text { Sector Agrario } \\
\text { Total Créditos }\end{array}$ & Miles mill. ptas. & Estructura & $\begin{array}{l}\text { Sector Agrario } \\
\text { Total Créditos }\end{array}$ & \\
\hline Entidades Oficiales de Crédito (1) .. & 145,88 & 12,95 & 7,70 & 24,99 & 13,00 & 11,11 & 17,13 \\
\hline 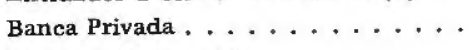 & 395,55 & 35,11 & 3,68 & 53,56 & 27,86 & 7,36 & 13,54 \\
\hline Cajas de Ahorro (2). . . . . . . & 186,42 & 16,55 & 4,96 & 27,60 & 14,35 & 8,08 & 14,80 \\
\hline Cajas Rurales (3) .......... & 396,23 & 35,18 & 100,00 & 85,36 & 44,41 & 100,00 & 21,54 \\
\hline 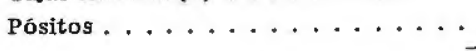 & 2,36 & 0,21 & 100,00 & 0,74 & 0,38 & 100,00 & 31,36 \\
\hline 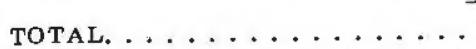 & $1.126,44$ & 100,00 & 7,09 & 192,25 & 100,00 & 12,29 & 17,07 \\
\hline
\end{tabular}

(1) Créditos directos, excepto la proporción SectorAgrario s/Total Créditos que incluye los créditos de provisión. No incluyen las subvenciones de los Organos Autónomos de la Administración del Estado cuya importancia es significativa, baste citar que en 1982 las concesiones del FORPPA, el SENPA, el IRYDA y las directas desde las distintas Direcciones Generales del Ministerio de Agricultura y Pesca fueron 60.800 millones de pesetas y que las dotaciones al FORPPA en los Presupuestos Generales del Estado para 1984 alcanzaron los 45.000 millones de pesetas.

(2) Existen grandes discrepancias entre los datos facilitados por la CECA y las Cuentas del Sector Agrario, MAPA, habiéndose tomado los primeros. Según el n. ${ }^{\circ} 9$ de las Cuentas del Sector Agrario la financiación al Sector Agrario de las Cajas de Ahorros ascendía en 1983 (avance) a 195.472 millones de pesetas sin incluir los préstamos de mediación.

(3) Los créditos concedidos con recursos propios significan el 91,72\% en el caso del total nacional y el 91,29 \% en Andalucía.

Fuente: Memorias de las EDC, Boletín Estadístico del CSB y del BE, las Estadísticas Agrarias del MAP A y Elaboración propia. 
En conjunto, el sistema crediticio dedica en Andalucía un 12,29 \% de sus colocaciones al sector agrario frente al 7,09\% a nivel nacional. Relacio. nando esta cifra $-12,29 \%-$ con la producción final agraria o con el valor añadido del sector se pone de manifiesto su insuficiencia. Las entidades crediticias se han desentendido con harta frecuencia del sector y así como han inventado y desarrollado fórmulas de captación de clientes mediante la oferta de servicios sofisticados para el sector industrial y el de servicios, no han sido todavía capaces de facilitar un servicio integral al agricultor.

En general, se puede afirmar que el Crédito de campaña está cubierto con creces. Tanto la Junta de Andalucía como el Banco Agrícola se han preocupado de que así fuera y las entidades crediticias privadas, por su parte, colaboran con agrado en unas colocaciones a corto plazo con elevadas garantías. Sin embargo, el crédito para inversiones está y ha estado profundamente desatendido ya que, se ha dirigido unidiricionalmente hacia una "pseudoindustrialización" del agro habiéndose acompañado de cuantiosas subvenciones cuyo resultado o cuyos beneficios escapan normalmente al agricultor medio al haberse centrado casi exclusivamente en la mecanización y en la comercialización.

Este, el agricultor medio, se enfrenta a un exceso de burocracia si pretende acceder al crédito oficial o a una carencia de garantías si se quiere dirigir al crédito privado. A grandes rasgos, las primeras entidades requieren avales o hipotecas y las segundas, garantías personales o avales, personales también. Ahora bien, esta primera barrera de entrada no sería difícil de atravesar si más allá de la misma se abriera un camino claro y fácil pero, nos encontramos con que las entidades crediticias suelen "ahogar" al agricultor con la premura de los vencimientos que aquilatan al máximo la estacionalidad de los cultivos. Por otra parte, existe una cierta especialización en las entidades dándose la paradoja de que a pesar de la misma entre todas ellas dejan ciertos segmentos del mercado sin cubrir. Asi, la Banca privada pudiera estar especializada en créditos a largo plazo y en explotaciones de tamaño superior a 200 $\mathrm{Ha}$., en cambio, las Cajas Rurales tienen la mayor parte de su clientela entre los agricultores con explotaciones inferiores a las $50 \mathrm{Ha}$. estando especialmente sesgadas hacia los créditos de campaña. La Banca oficial y las Cajas de Ahorro se dedican a todo un poco destacando sin embargo el medio y largo plazo de las primeras y el corto de las segundas.

En definitiva y a grandes rasgos se puede concluir que ese $35,53 \%$ de las explotaciones con tamaño entre $3 \mathrm{Ha}$. y $30 \mathrm{Ha}$. y que representan el $20,9 \%$ de la producción son los principales beneficiarios del $44,41 \%$ del crédito total concedido al sector a través de las Cajas Rurales y destinado, fundamentalmente, a créditos de campaña. 


\section{IMPORTANCIA DE LAS CAJAS RURALES EN EL SISTEMA BANCARIO ESPAÑOL}

Dados la escasa información y pocos conocimientos que normalmente existen sobre las Cajas Rurales, antes de centrarnos en la exposición de las Cajas Rurales de Andalucía, enmarcaremos a estas Entidades dentro del Sistema Bancario, tanto desde un punto de vista institucional como económicopatrimonial.

\subsection{Concepto, naturaleza y características de las Cajas Rurales}

"Es Cooperativa, aquella sociedad que realiza en régimen de empresa en común cualquier actividad económico-social lícita, para la mutua ayuda entre sus miembros y al servicio de éstos y de la comunidad" (Ley 52/1974 de 19 de Diciembre).

"Se clasifican como Cooperativas de Crédito las que tienen por exclusivo objeto social, servir las necesidades de financiación de las Entidades Cooperativas a ellas asociadas y a las de los socios de estas, pudiendo admitir imposiciones de fondos, así como realizar los servicios de banca necesarios y aquellos que sirvan para el mejor cumplimiento de los fines cooperativos, salvo los reservados expresamente a otros establecimientos de crédito, pudiendo realizar operaciones activas solo con sus socios y con los miembros singulares de las Entidades asociadas" (Real Decreto 2860/78 de 3 de Noviembre).

Las Cajas Rurales son Cooperativas de Crédito cuya finalidad es la intermediación financiera entre sus asociados fomentando y captando el ahorro y facilitando dichos recursos a sus asociados siempre que su destino sea de carácter agrario, mediante la garantía común, con bajo tipo de interés y condiciones de reembolso adaptadas a las necesidades de la producción agraria.

Las Cajas Rurales deben estar constituidas por Cooperativas del Campo, Sociedades Agrarias de Transformación y Cooperativas de Trabajo asociado para actividades exclusivamente agrarias así como por los miembros singulares de dichas Entidades, o bien por varias Cajas Rurales de ámbito Territorial inferior.

En este sentido la ley de Cooperativas andaluzas ha sido más progresista al establecer que pueden ser socios de esta clase de entidades además de las Cooperativas de crédito de ámbito territorial inferior, de las Cooperativas no crediticias que desarrollen su actividad preferentemente en el medio rural, y 
de las Sociedades Agrarias de Transformación, las personas físicas que desa rrollen actividades agrarias sin necesidad de pertenecer a Cooperativa alguna los trabajadores de la Caja siempre que hayan adquirido la condición de "so cios de trabajo", y las personas jurídicas de cualquier clase que desarroller actividades agrícolas.

En cuanto a la actividad que desarrollan las Cajas Rurales de créditc cooperativo presentan las siguientes limitaciones:

- Por su propia condición de Cooperativas, no pueden efectuar operaciones de activo mas que con sus socios, pudiendo recibir depósitos de terceros. La Ley de Sociedades Cooperativas de Andalucía, permite sin embargo efectuar operaciones activas con no socios hasta un $30 \%$ de los recursos totales de la Entidad.

- El ámbito de actuación es local comarcal o provincial, si bien con la firma del Convenio Individual de Asociación entre el B.C.A. y una gran parte de las Cajas Rurales, se amplía el marco de actuación del Grupo a un nivel nacional con posibles proyecciones a nivel internacional.

- Conviene mencionar aunque fuera del contexto legal de las Cooperativas de Crédito, y dentro de un núcleo de actuación muy reducido a las Secciones de Crédito de las Cooperativas. Estas, previa autorización del Banco de España, pueden constituir una Sección de Crédito que intermedie los flujos financieros de la Cooperativa y de sus socios, dentro de ciertas limitaciones como son: la apertura de depósitos a la vista ahorro y plazo solamente a los asociados a la Cooperativa a que pertenece, no pudiendo aceptar depósitos de terceros; y la concesión de préstamos o créditos destinados a la financiación propia de la Cooperativa en que se inserta, pudiendo facilitar recursos financieros a los socios por razón de tales operaciones y excepcionalmente por otras.

- Son instituciones monosectoriales, entidades especializadas sectorialmente al operar con socios agrarios.

- Y por último no pueden realizar todo el abanico de operaciones usuales en el sistema financiero: no pueden emitir Cédulas Hipotecarias, las no calificadas (Título que concede el Banco de España a las Cooperativas con más de 5 años de actuación y Recursos Propios superiores a 100 mill., que les permite participar en el crédito Oficial y redescuento y crédito del Banco de España) no pueden emitir certificados de depósito, no pueden admitir saldos en cuentas de ahorro bursátil, pesquero, cuenta fiscal de ahorro..., etc. 
Estas características condicionan la actividad de las Cajas Rurales dando lugar a la estacionalidad de sus recursos. El ahorro depositado procede fundamentalmente del sector agrario cuya producción tiene un carácter estacional, obteniéndose mayores recursos en los momentos en que el sector tiene menores necesidades de financiación y contrayéndose en las épocas de mayor demanda de crédito, lo que a su vez ha originado por un lado, el mantenimiento de un nivel de líquidez muy elevado para minimizar el riesgo de iliquidez y asegurar una rentabilidad aceptable desprovista de gestión y de riesgo y, por otro lado, la especialización en el corto plazo de los créditos concedidos. En este sentido cabe apuntar que un mecanismo de compensación a nivel regional evitaría al trasvase de los excedentes de tesorería a otros sectores al mismo tiempo que se aplicarían los recursos a la región de origen.

Otra peculiaridad negativa en las Cajas Rurales es tender por su propia naturaleza a la concentración de riesgos. Al efectuar operaciones activas solo con sus socios y dentro del medio rural, tienen un campo muy limitado para sus operaciones crediticias ello unido en muchos casos a una deficiente gestión bancaria y a vínculos de diversa índole ha originado una sobreacumulación de riesgos indebidamente garantizados que ha llevado a muchas Cajas Rurales a una situación de quiebra.

La propia limitación de las operaciones crediticias, con independencia de la evolución de la demanda de créditos, entre otros factores, reafirma a las Cajas para mantener unos niveles altos de tesorería.

\subsection{Grupo Asociado Banco de Crédito Agrícola-Cajas Rurales}

Las Cajas Rurales como hemos visto presentan: estacionalidad en sus recursos, una excesiva liquidez, escasa inversión crediticia altamente concentrada con fuertes riesgos y orientada fundamentalmente al corto plazo y dificultades con graves desequilibrios patrimoniales en algunos casos.

El B.C.A. por su parte adolece de una centralización del crédito con una red de oficinas muy reducida que dificulta el acceso del pequeño agricultor al crédito oficial (desconocido en muchos casos), está especializado en la financiación de inversiones agrarias, sus créditos son por tanto fundamentalmente a medio y largo plazo, y precisa fondos para su financiación —en tanto que la política del Gobierno sea ir reduciendo paulatinamente las dotaciones del Tesoro al Crédito Oficial, lo que unido a una liberación del sistema financiero mediante la reducción de coeficientes va a obligar al I.C.O. y a los Bancos Oficiales a captar directa o indirectamente recursos del mercado-.

Parecía pues lógica la simbiosis B.C.A./CAJAS RURALES que se materializó mediante la firma de un Acuerdo Marco el 19 de Julio de 1983 con el B.C.A. por 80 Cajas Rurales de las 127 existentes. La finalidad del Acuerdo es 
ampliar los canales financieros del sector agrario descentralizando la gestión aumentar los fondos financieros al sector, un Plan de Saneamiento para aque llas entidades con déficits patrimoniales, mejorar la distribución de riesgos, ? dotar al Banco de fondos necesarios para su financiación.

El Acuerdo-Marco contempla que todas las Cajas acogidas al mismo me diante Convenios individuales de asociación queden representadas en el Con sejo de Administración del Banco y se ajusten a una normativa contable I operativa determinada por el mismo quedando sometidas a una auditoría per manente. Al mismo tiempo se crean tres fondos con carácter solidario qu pretenden cubrir las posibles dificultades de liquidez - fondo de iliquide $z$ do tado con el 2,5\% de los pasivos computables para el coeficiente de cajade insolvencia - fondo de reserva para la Previsión de riesgos de insolvenci constituido por el $15 \%$ de los excedentes netos- y de tesorería mediante la aportación del $25 \%$ de la tesorería a un fondo común. Como contrapartide a estas condiciones, las Cajas Rurales podrían canalizar preferentemente los fondos del Crédito Oficial Agrario aunque sus destinatarios no sean socios -créditos de apoderamiento-.

Ahora bien, dada la situación de partida de algunas Cajas Rurales fué pre. ciso diseñar un Plan de Saneamiento para 19 Cajas y la Caja Rural Nacional previo a la firma de los Convenios individuales de asociación. Dicho Plan de Saneamiento aprobado por la Comisión Delegada del Gobierno para Asuntos Económicos en su reunión del 5 de Marzo de 1984, arbitraba una serie de ayudas que con cargo por un lado del Fondo de Garantía de Depósitos y, por otro, al Consorcio Nacional de Cajas Rurales provinciales, sirviera para equilibrar la situación patrimonial de dichas Cajas en un plazo de cinco años. Las ayudas facilitadas por el Fondo de Garantía de Depósitos han ascendido a 42.342.500.000 pesetas, mediante la concesión de préstamos sin interés a cinco años por un importe de 34.972.100.000 pesetas y el resto por compra de activos no funcionales. Cabe destacar que de este resto 3.583 .800 .000 pesetas corresponden a la Caja Rural Nacional. Por su parte, el Consorcio Nacional se comprometía al suministro de préstamos con interés del $8 \%$ y con vencimiento a cinco años por un total de 6.845.900.000 pesetas.

En Andalucía todas las Cajas Rurales Provinciales, excluida la de Cádiz que está a la espera del Segundo Plan de Saneamiento en estudio para cuatro o cinco Cajas por parte de la Comisión Delegada del Gobierno para Asuntos Económicos, han suscrito el Convenio Individual de Asociación junto con las Cajas comarcales y locales de Adamuz, Baena, Cañete de las Torres, La Carlota, Utrera y Ntra. Sra. del Rosario. 


\subsection{Las Cajas Rurales en el contexto del sistema bancario}

Determinadas las características más sobresalientes de las Cajas Rurales y del Grupo Asociado descendemos comparativamente por el Sistema Bancario a efectos de situar a las Cajas Rurales dentro del contexto nacional.

Desde 1977 se ha producido un proceso de homogeneización en el sistema bancario concretándose en el caso de las Cajas Rurales en el establecimiento de coeficientes obligatorios similares al resto del sistema, en el acceso a la financiación del Banco de España, en la limitación de las posibles concentraciones de riesgos iniciándose simultáneamente un proceso despolitizador de sus órganos de gobierno y gestión, y en la creación de un fondo de garantía de depositos. En definitiva se establece un mayor control efectivo por parte del Banco de España tratando de adecuar la dimensión de las Cajas a su ámbito de actuación e introduciéndolas dentro del sistema crediticio como un agente intermediario más.

$\mathrm{Si}$ analizamos comparativamente los grandes agregados de la estructura patrimonial de los distintos tipos de Entidades que componen el Sistema Bancario -Anexo n.o IV- vemos a través de los siguientes gráficos como se distribuyen los recursos, la tesorería, la inversión y el inmovilizado en función de las características intrínsecas del negocio bancario desarrollado por cada tipo de entidad.

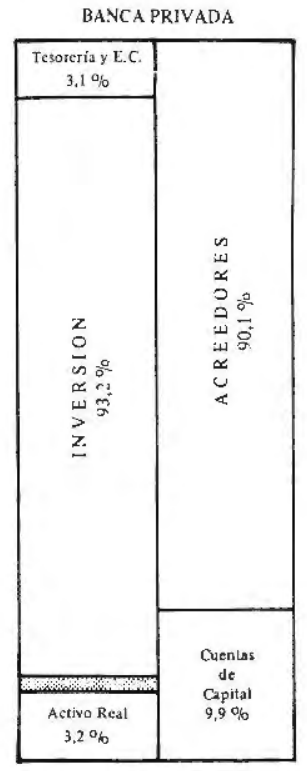

$0.5 \%$
CAJAS DE AHORRO

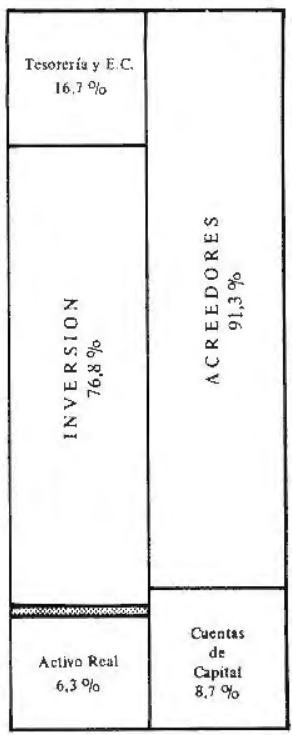

$0,2 \%$
CAJAS RURALES

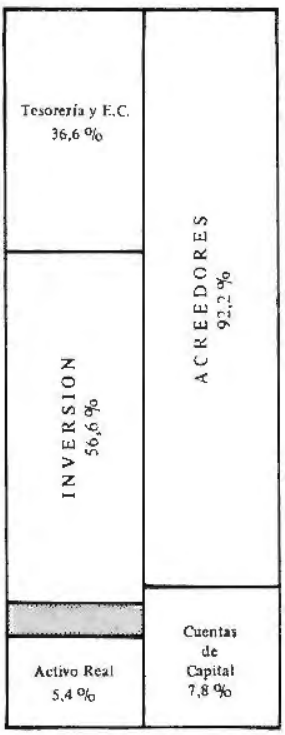

$1,4 \%$ 
Atendiendo a los recursos aportados y generados o captados por las dis tintas Entidades vemos que la proporción de recursos ajenos es ligerament superior en las Cajas Rurales. Las Cajas Rurales presentan unas cuentas de Capital minoradas por las cuantiosas pérdidas acumuladas (más de 30.006 mill. de pesetas) lo que repercute en una menor garantía con respecto a Ban cos y Cajas de Ahorros.

Mientra la banca privada tiene un bajo porcentaje de tesorería y recurso: facilitados a Entidades de Crédito -coeficiente de caja en el Banco de Espa ña - tomando recursos del crédito oficial (mediación) y del Sistema Banca. rio, las Cajas Rurales sin embargo, ceden recursos en una alta proporción a. B.E., a la Banca y al Banco de Crédito Agrícola principalmente. En una si. tuación intermedia se sitúan las Cajas de Ahorros, las cuales teniendo el mis. mo coeficiente de caja que la Banca (18\% pasivos computables) no obtiener recursos del B.E. cediendo, por tanto una mayor proporción de sus recursos que la Banca.

La Inversión varía en función inversa, decreciendo desde la Banca a las Cajas de Ahorro y a las Cajas Rurales. Mientras que tradicionalmente la Banca una vez cubiertas sus obligaciones de tesorería y de inversión obligatoria invierte libremente, las Cajas Rurales, una vez efectuadas sus inversiones finalistas colocan su tesorería excedente.

Por último, las Cajas Rurales mantienen un inmovilizado proporcionado con el resto del Sistema bancario, destacando la Banca con un inmovilizado relativamente inferior al no tener parte del mismo afecto a la Obra Benéfico Social o al Fondo de Educación y Obras Sociales como sucede con las Cajas de Ahorros o con las Cajas Rurales.

CUADRO II

DEPOSITOS DEL SECTOR PRIVADO

\begin{tabular}{lrrr}
\hline Años & Sistema Bancario & Cajas Rurales & $\%$ \\
\hline & & & \\
1972 & 2.990 .364 & $28.536(2)$ & 0,95 \\
1977 & 6.780 .919 & 161.171 & 2,37 \\
1982 & 15.038 .431 & 478.143 & 3,18 \\
1983 & 17.012 .094 & 556.337 & 3,27 \\
1984 & 19.055 .150 & 650.538 & 3,41 \\
\hline
\end{tabular}

(2) Cajas Rurales Provinciales. Fuente: Memoria Caja Rural Nacional.

Fuente: Boletín Estadístico del Banco de España y Elaboración propia. 
Por otro lado, examinando la evolución de su actividad durante los últimos años, encontramos que desde 1977 los recursos ajenos han crecido en un $303,6 \%$ frente al $181 \%$ experimentado por el sistema bancario en su conjunto. Es decir, han pasado de una cuota de mercado del 2,37 \% el 3,41 $\%$ en los últimos siete años pero hay que tener en cuenta que en 1972 sólo representaban el $0,95 \%$ del sistema bancario. Este rápido afianzamiento de las Cajas Rurales como captadoras de recursos ha tenido su contrapartida en el volumen de créditos concedidos con recursos propios cuyo aumento ha sido del $266 \%$ frente al 126,5\% del sistema bancario, pasando de una cuota de mercado del 1,6 \% al 2,7 \% del total créditos al sector privado del Sistema Bancario (Anexo n.० V).

CUADRO III

POSICION NETA DE LOS ACTIVOS Y PASIVOS CON EL SISTEMA BANCARIO

\begin{tabular}{|c|c|c|c|c|c|c|}
\hline & 1979 & 1980 & 1981 & 1982 & 1983 & 1984 \\
\hline \multicolumn{7}{|c|}{ Activos-Pasivos } \\
\hline financieros.......... & 109.130 & 118.987 & 138.735 & 128.691 & 137.697 & 108.674 \\
\hline \multicolumn{7}{|l|}{ \% sobre depósitos } \\
\hline sector privado . . . . . . & 36,7 & 36,3 & 34,5 & 26,9 & 24,7 & 16,7 \\
\hline
\end{tabular}

Fuente: B.E. y Elaboración propia.

Analizando los recursos que las Cajas Rurales ceden al Sistema Bancario vemos su evolución decreciente en los ultimos años, lo que redunda -salvando las pérdidas acumuladas - en una mayor inversión.

En el año 1984 el configurarse el Grupo Asociado B.C.A./Cajas Rurales, se trasvasan las posiciones de activo con el Sistema Bancario - Caja Rural Nacional- a posiciones de activo con el Crédito Oficial -Banco de Crédito Agrícola - resultando una misma posición neta global con el Sistema Crediticio -B.E., B.C.A. y Sistema Bancario- que en el año precedente.

En general, se puede afirmar que las Cajas Rurales a pesar de la crisis aflorada durante estos últimos tres años están fuertemente integradas tanto dentro del sistema crediticio como entre su clientela. El fuerte crecimiento de sus depósitos confirma lo segundo y sus posiciones de activo y pasivo en la Banca y en las Cajas de Ahorro, lo primero. 


\section{LAS CAJAS RURALES ANDALUZAS}

Las quince Cooperativas de Crédito andaluzas de las que se dispone información son agrarias, ocho son Cajas Rurales Provinciales y siete Cajas Rurales Comarcales y Locales, teniendo éstas poca incidencia al representar al 31-1284 el 1,6 \% de los depósitos totales de las Cajas Rurales Andaluzas.

Las cooperativas de crédito en Andalucía son relativamente recientes en contraposición con el arraigo tradicional del movimiento cooperativista en la región, ya que la primera Caja Rural que se funda es la Provincial de Jaén en 1957.

Su estructura patrimonial difiere ligeramente de la que examinamos a nivel nacional y ello por la importancia que han tenido y tienen a pesar de la crisis de 1982-83 y que suponemos seguirán manteniendo una vez superada la misma, en la intermediación del sector agrario.

Agregando en grandes partidas de balance el origen y aplicación de recursos de las Cajas Rurales Andaluzas (Anexo V) al igual que hicimos para el total Nacional, nos resulta la distribución siguiente:
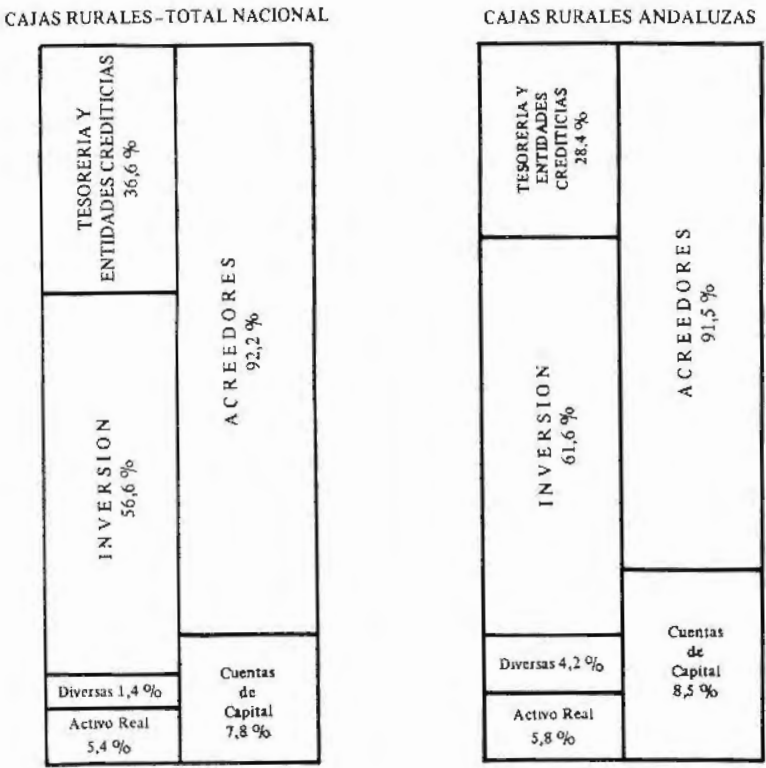

De la comparación de estructuras vemos que las Cajas Rurales Andaluzas mantienen mayor proporción de recursos propios y provisiones sobre recursos ajenos a pesar de las pérdidas acumuladas que aun mantienen a 31-12-84. 
Respecto a las partidas de Activo, las Cajas Andaluzas mantinen una proporción de sus recursos menor en el sistema bancario y similar en el Banco de España y en B.C.A., aplicando la diferencia en mayor inversión al sector privado.

Si bien hay que tener en cuenta que la partida "Inversión" incluye los morosos y dudosos que son ligeramente superiores en Andalucía así como las cuentas de Capital incluyen los Fondos para insolvencias (homogeneización con Boletín Estadístico B.E.) que a su vez son superiores con respecto al total Nacional. Descontando ambas partidas de la estructura patrimonial del conjunto de Cajas Rurales de Andalucía, ésta se acerca más a la del total Nacional, manteniendo no obstante la característica definitoria de menor tesorería y mayor inversión.

Las Cajas Rurales en Andalucía son muy heterogéneas para un análisis de conjunto y por ello, salvando la dimensión de las distintas Entidades, las agregamos en tres Grupos con objeto de poder realizar un análisis:

- El Grupo A incluiría aquéllas Cajas Rurales Provinciales con neto Patrimonial positivo: Almería, Huelva, Granada y Córdoba.

- En el Grupo B estarían aquellas Cajas Rurales Provinciales con neto Patrimonial negativo a saber: Sevilla, Jaén, Málaga y Cádiz, de las cuales las tres primeras están afectadas por el Plan de Saneamiento del B.C.A., habiendo quedado Cádiz en estudio para el segundo Plan de Saneamiento.

- Y el Grupo C engloba las Cajas Rurales Comarcales y Locales, es decir: "Ntra. Madre del Sol" de Adamuz, "Ntra. Sra. Guadalupe" de Baena, "Ntra. Sra. del Campo" de Cañete de las Torres, La Carlota, "Ntra. Sra. del Rosario" de Nueva Carteya, "San José" de Torredonjimeno, y de Utrera.

La característica más sobresaliente del ejercicio 1984 ha sido la fuerte disminución de la partida de morosos que no ha sido compensada con el incremento de la inversión realizada con recursos propios ( $\Delta$ del $3,2 \%$ ) por lo que el balance final es una aparente menor inversión crediticia. Del resto de las partidas del balance destacamos:

- El crecimiento del pasivo en un 15,84 \% para el conjunto de Cajas Ruraandaluzas frente el 17,73\% del total nacional. El comportamiento de las Cajas no ha sido, una vez más, uniforme y se ha notado algo la retracción de la clientela en aquellas cajas con dificultades. Así, mientras el grupo A supera en crecimiento a la media nacional con un $18,2 \%$, el grupo B se esfuerza en conseguir un aumento del $14,2 \%$ y el grupo $\mathrm{C}$ pierde cuota de mercado al mermar sus depósitos en un $12,2 \%$. 
- El fuerte incremento -un $96 \%$ - de la Tesorería y de los recursos cedi dos a otras Entidades de crédito. Este desproporcionado aumento se ha debido a la normalización de la Caja Rural Provincial de Jaén que tení: un riesgo con la Caja Rural Nacional de 8.000 millones de pesetas er. 1983. El B.C.A. al absorber los activos depositados en la misma además de los derivados del Convenio Individual de Asociación, partidas que re. presentan el $50 \%$ de la Tesorería de las Cajas, ha recibido recursos por valor de 30.000 millones de pesetas. Esta cifra se compara muy desfavo. rablemente para Andalucía con el volumen cedido de préstamos de me. diación que durante 1984 ha ascendido a unos 7.600 millones de pesetas, aproximadamente.

En cuanto a la cuenta de resultados, se ve afectada positivamente por la baja experiencia en la partida de deudores morosos y la consecuente liberación en el Fondo Especial para Insolvencias, no obstante, continua siendo un lastre el volumen de inmovilizado que mantienen, superior a la media nacional.

Esto es, especialmente, válido para las Cajas del Grupo B que habían llevado en años pasados una práctica de concentración de riesgos indebidamente garantizados cuya consecuencia fue un alto saldo de deudores morosos y dudosos para los que había que dotar en el Fondo de Insolvencias. A finales de 1983, el saldo de morosos en este grupo de Cajas representaban el $50 \%$ de la inversión crediticia, teniendo el Fondo dotado con $11.702,0$ millones de pesetas lo que significaría cubrir tan sólo un $50,7 \%$. Los resultados de explotación de dicho ejercicio arrojaron un déficit de 11.123,5 millones de pesetas que sumados a las pérdidas de ejercicios anteriores alcanzaron la cifra de 15.700,7 millones de pesetas, es decir, dos veces los Recursos Propios de las Entidades.

Durante 1984 se han realizado recuperaciones de inversión crediticia en mora a la vez que han surgido nuevas situaciones de mora por lo que la inversión en mora a finales de año era el 26,3 \% de la inversión crediticia contando con Dotaciones para Insolvencia el 66,7 \% de la misma. Los resultados positivos fueron de $6.927,1$ millones de pesetas en su mayor parte de las recuperaciones y los negativos por nuevas dotaciones ascendieron a 2.373,7 millones de pesetas acumulándose un total de pérdidas de 10.735,2 millones de pesetas las cuales se irán rebajando conforme se vayan produciendo recuperaciones de morosos con independencia de los resultados de explotación positivos que se pueden ir obteniendo y de las ayudas que puedan recibir por el Plan de Saneamiento.

En el grupo A los resultados positivos han aumentado con la excepción de Córdoba, por lo que se han reforzado sus Recursos propios. En este grupo los morosos a final de 1984 significaban el $8 \%$ del total inversión frente al $4 \%$ de 1983 . 
Por su parte, el Grupo de Cajas pequeñas conserva sus resultados positivos aunque con ligera disminución respecto al año anterior, reforzándose sus recursos propios sobre ajenos. El volumen de morosos no supera el $2 \%$ del total de inversión crediticia.

Un breve análisis de los diferentes conceptos que componen la cuenta de resultados para 1984 nos permite la siguiente radiografía de la cascada de márgenes.

1. La rentabilidad de los activos es superior a la media nacional en las Cajas pertenecientes a los grupos A y C. Respecto al grupo B ya nos hemos extendido anteriormente sobre el problema de los deudores en mora. La cartera de valores aunque arroja un bajo rendimiento no es muy significativa dada su pequeña cuantía.

2. El coste de los acreedores es inferior a la media nacional en los grupos A y $\mathrm{B}$, destacando el grupo $\mathrm{C}$ por su elevado coste.

3. Los costes de transformación son superiores a la media nacional en los grupos A y B. Resultando un margen de explotación sobre total pasivo superior a la media nacional para el conjunto de Caja, que componen el grupo $\mathrm{A}$ y $\mathrm{C}$ e inferior para el grupo $\mathrm{B}$.

Analizando los ratios de gestión acreedores por empleado activo y acreedores por oficina que sirven muy bien para situar a las Cajas dentro de su entorno, observamos para el conjunto un balance desfavorable. Así, el grupo A se encuentra en torno a la media nacional, el grupo B sensiblemente por debajo mientras que en el grupo $\mathrm{C}$ ambos ratios están por encima.

En síntesis, el ejercicio 1984 ha supuesto para todas las Cajas Rurales andaluzas su reorganización en función de su nueva condición de miembros de Grupo Asociado B.C.A./Cajas Rurales. Ha sido, pues, un año de ensayo o de estreno, como se le quiera denominar y los resultados de sus cuentas de explotación se han visto lógicamente afectados en mayor o menor medida pero, en conjunto, el balance final es alentador.

\section{CONSIDERACIONES FINALES}

Se puede decir que las Cajas Rurales andaluzas están atravesando el ecuador de su trayectoria ya que después de unos años de introducirse y romper en el mercado, con los consiguientes rápidos crecimientos en las partidas de activo y pasivo sigue, lógicamente, una reflexión sobre lo acontecido para poder rectificar los errores y afianzar los aciertos. Como hemos visto, las circunstancias tratado de forma desigual a las distintas Cajas pero todas ellas se 
encuentran en este momento bajo el manto del B.C.A. en cuanto que formar parte del Grupo asociado. El futuro se prevé pues con un cierto grado de confianza, confianza que puediera estar basada en dos pilares, a saber: la pro pia situación de mercado de las Cajas Rurales andaluzas y el apoyo y contro del B.C.A. en la gestión diaria de las mismas en la medida que represente un profesionalización de su papel como intermediarios financieros.

Las Cajas Rurales andaluzas tienen, todavía, que ganar cuota de mercadc dado que el grado de entendimiento del sector agrario andaluz se mantien por debajo de la media nacional. Ahora bien, avanzar en cuota de mercadc no es una tarea fácil y para ello las Cajas Rurales deberán afianzar y, algunas de ellas, recuperar su imagen. Es evidente, que el Grupo asociado está con. tribuyendo a este lavado de imagen pero éste no será suficiente si el servicio prestado diariamente no es eficaz. Rendir eficaz el servicio significa: rigor técnico y eficiencia en sus órganos rectores y dirección, implantar la mecanización en las oficinas, liberar activos improductivos, crear instrumentos váli. dos de captación de pasivo, realizar estudios de mercado interior y exterior para acercar la oferta de servicios a los potenciales clientes de activo, desarrollar una sociedad de servicios a nivel regional que les permita acceder al mercado financiero directamente con un mínimo de costes y poner el mismo a disposición de los diversos clientes..., etc. En una palabra, diseñar una actividad crediticia moderna y prestar un servicio integral al agricultor.

No cabe duda que la simbiosis con el B.C.A. participa de esta idea de fondo y no se puede negar que hasta el momento ha sido positiva. De entrada, la tutela del B.C.A. ha servido para que las distintas Cajas supieran cual era su situación patrimonial real, las Cajas en dificultades han tenido acceso a un plan de Saneamiento cuya viabilidad se está demostrando a medida que avanza el mismo y no se ha perdido la imagen de marca aunque se haya visto empañada en algunos momentos. Ahora bien, el B.C.A. tampoco está exento de críticas puesto que no todo iban a ser parabienes en este singular maridaje al que en alguna medida se han visto obligadas las Cajas como contrapartida a servir como canal preferencial del crédito oficial. De momento, y pasará tiempo hasta que cambie la forma de pensar en los responsables de los organismos vinculados directamente a la Administración del Estado, al B.C.A. se le puede tachar de llevar una política o una estrategia excesivamente centralizada sin tener muy en cuenta al Estado de las Autonomías. Y, concretamente en Andalucía, ha sido la Junta quien ha dado los primeros pasos para modernizar a las Cajas Rurales - la Ley de Cooperativas puede ser y de hecho está siendo modelo progresista-, para acercar la financiación al cliente - los Convenios firmados por la Consejería de Agricultura para préstamos de campaña y de circulante y el Convenio con A.S.I.C.A. son buena prueba-, - las circulares de obligado cumplimiento de la Direción General de Política Financiera-... 
En definitiva, conscientes las distintas administraciones de la importancia que tiene y del grado de desarrollo que debe alcanzar el intermediario financiero "Cajas Rurales" en el contexto regional andaluz, se están preocupando por crear o mejorar las condiciones institucionales que lo permitan.

ANEXO I

MACROMAGNITUDES DEL SECTOR AGRARIO (1982)

\begin{tabular}{|c|c|c|c|}
\hline & $\begin{array}{c}\text { España } \\
\text { (Miles mill. ptas.) }\end{array}$ & $\begin{array}{c}\text { Andalucía } \\
\text { (Miles mill. ptas.) }\end{array}$ & $\begin{array}{c}\% \\
\text { respecto España }\end{array}$ \\
\hline Produccion total $\ldots \ldots \ldots$ & $2.311,94$ & 417,05 & 18,04 \\
\hline Reempleo . . . . . . . . & 372,16 & 48,24 & 12,96 \\
\hline Produccion final. . . . . . . & $1.939,78$ & 368,81 & 19,01 \\
\hline 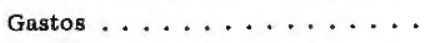 & 817,89 & 112,42 & 13,74 \\
\hline U.A.B. p. mercado $\ldots \ldots \ldots$ & $1.121,89$ & 256,39 & 22,85 \\
\hline Subvención a la explotación. . . . & 26,60 & 7,31 & 27,48 \\
\hline U.A.B. Coste factores. . . . . . & $1.148,49$ & 263,70 & 22,96 \\
\hline Amortizaciones . . . . . . & 154,92 & 22,30 & 14,39 \\
\hline Renta Agraria . . . . . . . & 993,57 & 241,47 & 24,30 \\
\hline
\end{tabular}

Fuente: Las Cuentas del Sector Agrario, n. ${ }^{\circ}$ 9. M.A.P.A.

ANEXO II

ENTIDADES OFICIALES DE CREDITO

Crédito concedido al Sector Agrario en Andalucía (1984)

\begin{tabular}{|c|c|c|c|c|c|}
\hline \multirow{2}{*}{ Entidades Oficiales de Crédito } & \multicolumn{2}{|c|}{ Espeña (1) } & \multicolumn{2}{|c|}{ Andalucía (2) } & \multirow{2}{*}{$(2) /(1)$} \\
\hline & Mill. ptas. & Estruc. & Mill. ptas. & Estruc. & \\
\hline B.C.A. $\ldots \ldots \ldots \ldots \ldots \ldots$ & 220.205 & 100,00 & 42.828 & 100,00 & 19,45 \\
\hline - Provisión a colaboradores...... & 80.861 & 36,72 & 18.469 & 42,12 & 22,84 \\
\hline 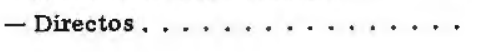 & 139.344 & 63,28 & 24.359 & 56,88 & 17,48 \\
\hline B.H.E. $\ldots \ldots \ldots \ldots \ldots \ldots$ & 820.671 & 100,00 & 235.490 & - & 28,70 \\
\hline -Mejoras de fincas rústicas . . . . . & 2.248 & 0,27 & 636 & - & - \\
\hline c.s.P.............. & 15.495 & 100,00 & 3.474 & - & 22,42 \\
\hline I.c.o.. $\ldots \ldots \ldots \ldots$ & 117.790 & 100,00 & s.d. & - & s.d. \\
\hline - Fondo d. ${ }^{\circ}$ ganadero . . . . . & 4.294 & 3,65 & s.d. & - & s.d. \\
\hline TOTAL Crédito al Sector Agrario. . . & 226.747 & 7,70 & 43.464 & 11,11 & 19,16 \\
\hline TOTAL Crédito de E.O.C. . . . . & 2.945 .527 & 100,00 & 391.157 & 100,00 & 13,28 \\
\hline
\end{tabular}

Fuente: Memoria I.C.O, para 1984. Memoria B.C.A para 1984. Memoria B.H.E. para 1984 y Boletín Estadístico B.E. 
ANEXO III

ESTRUCTURA PATRIMONIAL POR GRANDES AGREGADOS

\begin{tabular}{|c|c|c|c|c|c|c|}
\hline & \multicolumn{2}{|c|}{ Banca Privada } & \multicolumn{2}{|c|}{ Cajas de Ahorro } & \multicolumn{2}{|c|}{ Cajas Rurales } \\
\hline & Mill. ptas. & $\begin{array}{c}\text { Estructura } \\
(\%)\end{array}$ & Mill. ptas. & $\begin{array}{c}\text { Estructura } \\
(\%)\end{array}$ & Mill. ptas. & $\begin{array}{c}\text { Estructura } \\
(\%)\end{array}$ \\
\hline \multicolumn{7}{|l|}{ Tesorería y Entidades de Crédito } \\
\hline (neto) $(1) \ldots \ldots \ldots \ldots$ & 565.846 & 3,1 & 1.495 .474 & 16,7 & 269.898 & 36,6 \\
\hline Inversión . . . . . . . . . . & 17.169 .704 & 93,2 & 6.875 .750 & 76,8 & 416.941 & 56,6 \\
\hline Diversas (neto) $\ldots \ldots \ldots \ldots$ & 90.407 & 0,5 & 15.713 & 0,2 & 10.110 & 1,4 \\
\hline 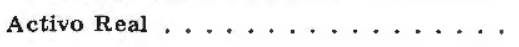 & 586.522 & 3,2 & 565.507 & 6,3 & 39.562 & 5,4 \\
\hline 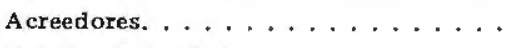 & 16.587 .384 & 90,1 & 8.170 .730 & 91,3 & 678.823 & 92,2 \\
\hline Cuentas de Capital $\ldots \ldots \ldots \ldots$ & 1.825 .095 & 9,9 & 786.714 & 8,7 & 57.688 & 7,8 \\
\hline TOTAL ACTIVO Y PASIVO ....... & 18.412 .479 & 100,0 & 8.952 .444 & 100,0 & 736.511 & 100,0 \\
\hline
\end{tabular}

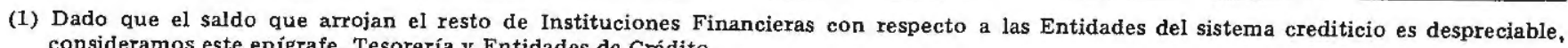
consideramos este epígrafe, Tesorería y Entidades de Crédito.

Fuente: Boletín Estadístico B.E. y Elaboración propia.

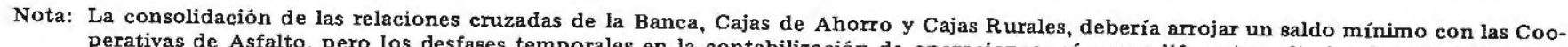
perativas de Asfalto, pero los desfases temporales en la contabilización de operaciones asi como diferentes criterios de contabilización, hace que se origine un descuadre superior a los 100.000 millones de pesetas, el B.E. está trabajando para lograr homogeneizar las series. 
ANEXO IV

BALANCE DEL SISTEMA BANCARIO AL 31-XII-84

(Millones de pesetas)

\begin{tabular}{|c|c|c|c|}
\hline ACTIVO & Banca Privada & Cajas de Ahorro & Cajas Rurales \\
\hline \multicolumn{4}{|l|}{ Instituciones Financieras (neto) } \\
\hline - В.E. . . . . . . . . . . & 1.150 .220 & 1.334 .036 & 60.574 \\
\hline -Sistema Bancario. . . . . . . . . . & - & 180.528 & 108.674 \\
\hline - Crédito oficial ........... & - & - & 100.119 \\
\hline - Resto $\ldots \ldots \ldots \ldots \ldots \ldots$ & 8.422 & 6.031 & 531 \\
\hline \multicolumn{4}{|l|}{ Inversión } \\
\hline - Sector Público . . . . . . . . . & 3.595 .777 & 2.080 .149 & 15.218 \\
\hline - Sector Privado . . . . . . . . & 10.605 .827 & 4.726 .721 & 401.679 \\
\hline - Sector Exterior. . . . . . . . & 2.968 .100 & 68.880 & 44 \\
\hline Diversas Neto $\ldots \ldots \ldots \ldots \ldots$ & 90.407 & 15.713 & 10.110 \\
\hline Activo Real $\ldots \ldots \ldots \ldots \ldots \ldots$ & 586.522 & 565.507 & 39.562 \\
\hline TOTAL ACTIVO . . . . . . . . . . & 19.005 .522 & 8.977 .565 & 736.511 \\
\hline \multicolumn{4}{|l|}{ PASIVO } \\
\hline \multicolumn{4}{|l|}{ Instituciones Financieras (neto) } \\
\hline - В.E. . . . . . . . . & - & - & - \\
\hline - Sistema Bancario. . . . . . . . & 178.065 & - & - \\
\hline - Crédito oficial $\ldots \ldots \ldots \ldots \ldots$ & 414.751 & 25.121 & - \\
\hline- Resto $\ldots \ldots \ldots \ldots \ldots$ & - & - & - \\
\hline \multicolumn{4}{|l|}{ Acreedores } \\
\hline - Sector Público ............ & 340.159 & 352.010 & 11.680 \\
\hline - Sector Privado . . . . . . . . & $12.970,286$ & 7.711 .851 & 666.979 \\
\hline - Sector Exterior . . . . . . . . . & 3.276 .939 & 106.869 & 164 \\
\hline Diversas Neto $\ldots \ldots \ldots \ldots \ldots$ & - & - & - \\
\hline Cuentas de Capital $\ldots \ldots \ldots \ldots$ & 1.825 .095 & 781.714 & 57.688 \\
\hline TOTAL PASIVO $\ldots \ldots \ldots \ldots \ldots$ & 19.005 .295 & 8.977 .565 & 736.511 \\
\hline
\end{tabular}


ANEXO $\mathrm{V}$

CREDITOS AL SECTOR PRIVADO

\begin{tabular}{|c|c|c|c|c|c|c|c|}
\hline \multirow{2}{*}{ Años } & \multicolumn{2}{|c|}{ Por Instituciones Prestamistas } & \multicolumn{2}{|c|}{ De Provisión } & \multicolumn{2}{|c|}{ Por Fuentes Financieras } & \multirow{2}{*}{$\%$} \\
\hline & Sistema Bancario & Cajas Rurales & Sistema Bancario (1) & Cajas Rurales (2) & Sistema Bancario & Cajas Rurales & \\
\hline 1972 & 2.006 .700 & 22.612 & 23.200 & 5.662 & 1.983 .500 & 16.950 & 0,8 \\
\hline 1977 & 6.064 .706 & 113.195 & 127.785 & 13.935 & 5.936 .921 & 99.260 & 1,6 \\
\hline 1982 & 12.848 .637 & 366.083 & 506.326 & 28.361 & 12.342 .311 & 337.722 & 2,7 \\
\hline 1983 & 14.214 .391 & 381.370 & 644.412 & 27.527 & 13.833 .021 & 353.843 & 2,5 \\
\hline 1984 & 14.160 .843 & 396.235 & 715.921 & 32.824 & 13.444 .922 & 363.411 & 2,7 \\
\hline
\end{tabular}

(1) Incluye créditos de provisión ICO. BCA y $\mathrm{BCE}$.

(2) BCA.

Fuente: Boletín Estadistico de España. Memoria ICO. Memoria BCA. Memoria CR UNA y Elaboración propia. 


\section{ANEXO VI \\ CAIAS RURALES ANDALUZAS SITUACION AL 31-XII-84}

\begin{tabular}{|c|c|c|}
\hline ACTIVO & Millones de pesetas & $\begin{array}{c}\text { Estructura } \\
(\%)\end{array}$ \\
\hline Instituciones Financieras (neto) $\ldots \ldots \ldots \ldots$ & $43,595,8$ & 28,4 \\
\hline - B.E. (activos líquidos) . . . . . . . & $12.858,4$ & \\
\hline - Sistema Bancario. . . . . . . . . . . . & $8.094,5$ & \\
\hline - Crédito oficial . . . . . . . . . . & $21.840,8$ & \\
\hline - Resto Entidades financieras y otras cuentas. . & 802,1 & \\
\hline Inversion $\ldots \ldots \ldots \ldots \ldots \ldots$ & $94.383,1$ & 61,6 \\
\hline - Sector Privado ................ & $86.869,2$ & \\
\hline - Sector Público $\ldots \ldots \ldots \ldots \ldots \ldots$ & $7.513,9$ & \\
\hline - Sector Exterior. . . . . . . . . . & - & \\
\hline Diversas (neto) $\ldots \ldots \ldots \ldots \ldots$ & $6.465,3$ & 4,2 \\
\hline Activo Real $\ldots \ldots \ldots \ldots \ldots \ldots$ & $8.899,4$ & 5,8 \\
\hline 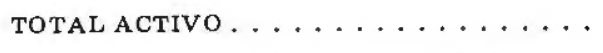 & $153.343,6$ & 100 \\
\hline PASIVO & & \\
\hline Acreedores. . . . . . . . . . . & $140.345,7$ & 91,5 \\
\hline- Sector Privado $\ldots \ldots \ldots \ldots \ldots$ & 136.981 .4 & \\
\hline - Sector Público $\ldots \ldots \ldots \ldots \ldots \ldots$ & $3.364,3$ & \\
\hline - Sector Exterior. . . . . . . . . . & - & \\
\hline Cuentas de Capital (1) . . . . . . . & $12.997,9$ & 8,5 \\
\hline TOTAL PASIVO $\ldots \ldots \ldots \ldots \ldots$ & $153.343,6$ & 100 \\
\hline
\end{tabular}

(1) Incluy e: Dotaciones a capital. 
CAJAS RURALES ANDALUZAS

(En millones de pesetas)

\begin{tabular}{|c|c|c|c|c|c|c|c|c|}
\hline \multirow{2}{*}{ ACTIVO } & \multicolumn{2}{|c|}{ Grupo A } & \multicolumn{2}{|c|}{ Grupo B } & \multicolumn{2}{|c|}{ Grupo C } & \multicolumn{2}{|c|}{ Total } \\
\hline & 1983 & 1984 & 1983 & 1984 & 1983 & 1984 & 1983 & 1984 \\
\hline Tesorería y E.C. (neto). . . . . . & $18.981,8$ & $24.730,4$ & 1.925 & $17.959,5$ & $1.340,1$ & 905,9 & $22.246,9$ & $43.595,8$ \\
\hline - Caja y Banco de España . . . . . & 6.613 & $7.324,4$ & 2.354 & $5.370,1$ & 286,2 & 163,9 & $9.253,2$ & $12.858,4$ \\
\hline - Sistema Bancario $(1) \ldots \ldots$ & 11.805 & $3.419,5$ & 502.4 & $4.247,5$ & $1.019,5$ & 427,9 & $13.326,9$ & $8.094,5$ \\
\hline - В.С.A. . . . . . & -2.195 & $13.514,5$ & $-1.222,7$ & $8.037,1$ & 28,5 & 289,2 & $-3.389,2$ & $21.840,8$ \\
\hline$\rightarrow$ Otras Cuentas. . . . . . & $2.758,8$ & 472,1 & 291,3 & 304,8 & 5,9 & 25,2 & 3.056 & 802,1 \\
\hline Inversiones Crediticias . . . . . & $45.386,9$ & $50.699,8$ & 45.823 & $33.304,8$ & $1.180,6$ & $1.354,8$ & $92.390,5$ & $85.359,4$ \\
\hline - Préstamos y Créditos. . . . . . & $41.128,2$ & $42.657,8$ & $20.801,3$ & $21.118,2$ & $1.168,7$ & $1.303,9$ & $63.098,2$ & $65.079,9$ \\
\hline - Ptmos. Ctos. mediación . . . . & $2.448,5$ & $3.977,7$ & $1.935,5$ & 3.438 & - & 26,3 & 4.384 & 7.432 \\
\hline - Morosos y dudosos. . . . . . & $1.810,2$ & $4.064,3$ & $23.086,2$ & $8.758,6$ & 11,9 & 24,6 & $28.908,3$ & $12.847,5$ \\
\hline Cartera de Títulos. ....... & 5.947 & $6.801,5$ & $2.381,6$ & $1.999,8$ & 227,5 & 222,4 & $8.556,1$ & $9.023,7$ \\
\hline 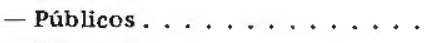 & $4.429,4$ & $5.701,3$ & $1.491,5$ & $1.599,4$ & 207 & 213,2 & $6.127,9$ & $7.513,9$ \\
\hline 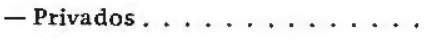 & $1.517,6$ & $1.100,2$ & 890,1 & 400,4 & 20,5 & 9,2 & $2.428,2$ & $1.509,8$ \\
\hline Activo Real $\ldots \ldots \ldots \ldots$ & $3.210,4$ & $4.176,1$ & $4.156,3$ & $4.579,8$ & 119,5 & 143,5 & $7.468,2$ & $8.899,4$ \\
\hline 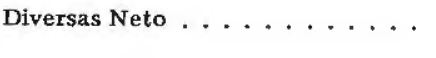 & $1.385,1$ & $2.903,8$ & $1.189,8$ & $3.499,2$ & 62,2 & 62,3 & $3.319,1$ & $6.465,3$ \\
\hline 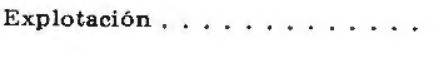 & - & 198,1 & $11.123,5$ & $2.175,6$ & - & - & $11.123,5$ & $2.373,7$ \\
\hline Pérdidas Ejercicios anteriores ... & - & - & $4.577,2$ & $15.486,7$ & - & - & $4.577,2$ & $15.486,7$ \\
\hline 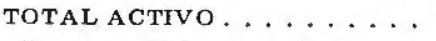 & $74.911,2$ & $89,509,7$ & $71.856,4$ & $79,005,4$ & $2.929,9$ & $2.688,9$ & $149.697,5$ & 171.204 \\
\hline
\end{tabular}

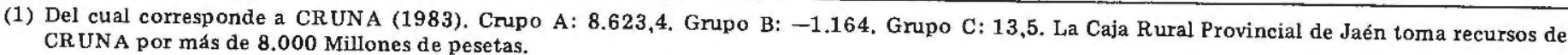


ANEXO VII (Continuación)

\begin{tabular}{|c|c|c|c|c|c|c|c|c|}
\hline \multirow{2}{*}{ PASIVO } & \multicolumn{2}{|c|}{ Grupo A } & \multicolumn{2}{|c|}{ Grupo B } & \multicolumn{2}{|c|}{ Grupo C } & \multicolumn{2}{|c|}{ Total } \\
\hline & 1983 & 1984 & 1983 & 1984 & 1983 & 1984 & 1983 & 1984 \\
\hline Cuentas Capital . . . . . . . & $6.317,6$ & $8.312,7$ & $6.707,3$ & $5.625,5$ & 262,2 & 332,2 & $13.287,2$ & $14.270,4$ \\
\hline \multicolumn{9}{|l|}{ Fondo Especial Insolvencias } \\
\hline Acredores $\ldots \ldots \ldots \ldots$ & $1.495,1$ & $1.960,8$ & $11.702,8$ & $5.843,7$ & 9,8 & 15,7 & $13.207,7$ & $7.820,2$ \\
\hline Acreedores. . . . . . . . . & $65.442,5$ & $77.472,8$ & $53,446,3$ & $60.609,1$ & $2.565,6$ & $2.263,8$ & $121.454,4$ & $140.345,7$ \\
\hline - Sector Privado . . . . . . . & $63.833,4$ & $74.824,4$ & $51.718,2$ & $57.631,6$ & 2.472 & $2.193,7$ & $118.023,6$ & $134.649,7$ \\
\hline - Sector Público . . . . . . . . & 637,8 & $1.383,6$ & 450,5 & $1.948,6$ & 26,4 & 32,1 & $1.114,7$ & $3.364,3$ \\
\hline 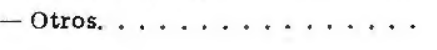 & 971,3 & $1.264,8$ & $1.277,6$ & $1.028,9$ & 67,2 & 38 & $2.316,1$ & $2.331,7$ \\
\hline Explotacion $\ldots \ldots \ldots \ldots$ & 1.656 & $1.763,4$ & - & $6.927,1$ & 92,2 & 77,2 & $1.748,2$ & $8.767,7$ \\
\hline TOTAL PASIVO . . . . . . . . & $74.911,2$ & $89.509,7$ & $71.856,4$ & $79.905,4$ & $2.929,9$ & $2.688,9$ & $149.675,5$ & $171.204,0$ \\
\hline
\end{tabular}

Fuente: Banco de España y Elaboración propia,

Grupo A: Cajas Rurales Provinciales de: Almería, Huelva, Granada y Córdoba.

Grupo B: Cajas Rurales Provinciales de: Sevilla, Jaén, Málaga y Cádiz.

Grupo C: Cajas Rurales Comarcales y Locales de: Baena, Nueva Carteya, Cañete de las Torres, Adamuz, La Carlota (Córdoba), Torredonjimeno (Jaén), Utrera (Sevilla). 